\title{
Cardiac MRI visualization for ventricular tachycardia ablation
}

\author{
Corine J. Godeschalk-Slagboom • Rob J. van der Geest • \\ Katja Zeppenfeld · Charl P. Botha
}

Received: 28 December 2011 / Accepted: 11 June 2012 / Published online: 3 July 2012

(C) The Author(s) 2012. This article is published with open access at Springerlink.com

\begin{abstract}
Objective The integrated visualization of cardiac MRI during a ventricular tachycardia (VT) mapping and ablation procedure would provide improved catheter guidance and tissue assessment. We developed a system for and explored the added value of simultaneous visualization of intracardiac voltage measurements and MRI-derived myocardial scar information during VT ablation procedures.

Method We propose the use of a synchronized 3D and 2D view. In 3D, the catheter will be guided optimally by assessing 3D scar characteristics and its relation to the ventricular anatomy. In 2D, a detailed assessment of the tissue can be made. We developed several 3D visualization techniques, including volume rendering of the scar and myocardial surfaces colored according to the voltage measurements. We also visualized context structures in the heart. For the 2D view, we proposed showing three adjacent slices simultaneously. To link the 3D with the 2D view, we added a linking plane and linking contours; the slice level shown in the 2D view is indicated in the $3 \mathrm{D}$ view.
\end{abstract}

C. J. Godeschalk-Slagboom · R. J. van der Geest $(\varangle) \cdot$ C. P. Botha Division of Image Processing, Department of Radiology, Leiden University Medical Center, Leiden, The Netherlands

e-mail: rvdgeest@lumc.nl; R.J.van_der_Geest@lumc.nl

C. J. Godeschalk-Slagboom

Biomedical Engineering, Delft University of Technology, Delft, The Netherlands

K. Zeppenfeld

Department of Cardiology, Leiden University Medical Center, Leiden, The Netherlands

\section{P. Botha}

Computer Graphics and Visualization, Department of Intelligent Systems, Delft University of Technology, Delft, The Netherlands
Results We evaluated our method via a case study during which we simulated the visual environment of an ablation procedure. The MRI-based volume rendering of scar tissue and the linking between the $3 \mathrm{D}$ and $2 \mathrm{D}$ views were both positively received. However, the visualization of the voltage measurements was found to be hard to interpret, partly due to the perceptually suitable but non-standard colormap. Conclusions Based on this study, we can conclude that our approach of displaying MRI data and integrating it with voltage measurements has potential to improve VT ablation procedures.

Keywords Multi-modal visualization · Cardiac MRI · Ventricular tachycardia $\cdot$ Catheter ablation

\section{Introduction}

Visualization of myocardial tissue properties plays an important role in catheter ablation procedures for the treatment of ventricular tachycardia (VT). Traditionally, myocardial voltage measurements are the basis for the ablation [1] but various studies have demonstrated the correlation between late enhancement (LE) magnetic resonance imaging (MRI) and voltage mapping [2,3]. Since the LE-MRI data is available prior to the ablation procedure, it has great potential to improve the procedure as the operator can focus immediately on the area of interest, thereby reducing procedure time and radiation exposure. In addition, MR reflects biological information different from voltage mapping and it can enable detailed myocardial tissue assessment. This study concerns the combined visualization of MR data and voltage measurements for the guidance of VT ablation procedures.

The method we propose consists of a 3D multi-modal visualization for global overview and guiding the catheter 
and a 2D slice view for detailed inspection of the MRI data. The views are synchronized, and through specially selected visual elements assist the user in matching the scenes that are differently represented in the two views. For the 3D view we developed several visualizations: We use volume rendering to show the scar tissue and we present two techniques to visualize unstructured voltage measurements over the heart surface. The linking with a $2 \mathrm{D}$ view offers a practical visualization environment. We evaluated the use of these visualizations for catheter ablation via a case study; while simulating an ablation procedure we had a focused interview with a cardiologist who is an expert in clinical VT ablations.

The main contribution of this paper is that it is the first practical investigation of the combined display of pre-procedural MRI data together with intra-procedural voltage measurements in order to improve VT ablation procedures. In addition, from the case study evaluation, increased insight was obtained into the specific needs of a cardiologist during VT ablation, which can be used in further research.

\section{Background}

Ventricular tachycardia is a life-threatening arrhythmia originating from the ventricles of the heart. The most common mechanism is scar-related re-entry. The main cause of myocardial scar is a former myocardial infarction $[1,4]$. The presence of inhomogeneous myocardial scar can lead to regions of slow conduction and regions of conduction block, which are preconditions for re-entry tachycardias $[1,5,6]$. The goal of radiofrequency ablation is to interrupt the critical slowconducting part of the re-entry circuit. Conventionally, catheter mapping to identify successful ablation sites is based on electrogram characteristics. In particular, low voltages electrograms correspond to scar tissue [2,3].

It is important to realize that during the mapping procedure, the operator who navigates the catheter is not able to control simultaneously the user interface of the visualization. The user verbally instructs an assistant to interact with the visualization environment. This should be taken into account when developing new visualization techniques.

LE-MRI is currently the gold standard for assessment of scar tissue in the myocardium. Due to slower wash-out of the contrast agent Gadolinium, scar regions appear bright on the MRI $[1,2,5,7]$. To take into account the heterogeneity of scar, the scar region on MRI can be subdivided into core infarct and gray zone, being the brightest parts and the darker parts of the scar, respectively [8-10]. Gray zone is mostly present at the boundaries of the scar. Making the scar information from the MRI available within the ablation environment could help reduce procedure time. Dickfeld et al. [11] have already showed the feasibility of 3D MRI scar reconstructions to facilitate VT ablations. The next step is the search for the best visual integration of the MRI scar information and the voltage measurements, which is the research topic described in this paper.

\section{Requirements}

Based on this background information, together with our observations during several ablation procedures and the outcome of interviews with the clinicians involved in the ablation procedures, we set the following requirements for our visualization system:

- The interface should integrate both MRI and voltage measurements, so that structural information (anatomy, scar geometry) can be effectively combined with functional information (voltage measurements), in order to facilitate the cardiologists decision-making during the VT ablation.

- The system should provide overview of the heart characteristics, to improve catheter guidance based on a quick localization of critical (scar) areas.

- The system should provide detailed MRI information for user-defined regions, to assess the myocardial tissue during the procedure.

- The interface should automatically adapt to the intention of the user to reduce the need of user interaction.

- The development of the system should not be restricted by the limitations of the currently used ablation systems, in order to investigate all possible ways of displaying MRI data together with voltage measurements.

\section{Related work}

In previous work on the visualization of MR defined scar performed by Wijnmaalen et al. [12] and Codreanu et al. [13], they computed the regional scar transmurality, that is, the local amount of scar tissue relative to the wall thickness, and projected it on the MR-defined heart wall surface by color encoding. Although this parameter correlates well with the voltage measurements, it provides little help in inferring the 3D morphology of the scar. Based on this type of visualization, also the detail of the 3D volume has been reduced to a 2D surface, resulting in loss of useful information. An alternative approach to the representation of scar transmurality was presented by Termeer et al. [14]. He suggests the use of a volumetric bull's eye plot in which the distribution of scar in the heart wall can be assessed at a glance. In that representation however, the real anatomical shape of the heart is not visible, which makes this technique inappropriate for catheter navigation purposes.

Oeltze et al. [15] developed several visualization techniques to represent myocardial perfusion, viability (healthy tissue versus scar tissue) and contractility information in a single view. To realize this they used several building blocks: a surface rendering of the scar within a transparent surface of 
the heart wall, depth isolines or a color-coded scar surface to represent the distance of the scar from the epicardium, combined with glyphs that represent the perfusion parameters. In addition to the scar morphology information provided by this representation, our method aims to visualize tissue properties from inside the scar tissue as well. In our multi-modal visualization, the idea of scar representation in combination with the transparent heart wall surfaces is inspired by this work of Oeltze et al.

Termeer [16] presented a comprehensive cardiac visualization in which viability, perfusion and thickening of the heart wall are brought together. He uses color coding, glyphs and an overlay in a striped pattern to visualize the individual parameters. However, the scar representation by a striped pattern overlay provides insufficient detail for the purpose of our visualization.

\section{Overview}

This paper is organized as follows: First, we describe the method we propose and how we implemented it in MeVisLab. We evaluated our method through a case study, which is described in the subsequent section. The final section contains conclusions and recommendations for further work.

\section{Method}

To provide catheter guidance as well as myocardial tissue assessment by LE-MRI data, we propose to use a configurable $3 \mathrm{D}$ visualization in combination with a $2 \mathrm{D}$ slice view. The main purpose of the 3D view is to provide an overview enabling the user to immediately see the scar distribution. The main purpose of the $2 \mathrm{D}$ slice view is to provide, for a selected region, all the details available from the MRI to assess the myocardial tissue. By linking the 2D and 3D view, the user can take full advantage of both views simultaneously. Figure 1 shows an impression of the visualization environment.

In this section, we first discuss the preprocessing steps and then the 3D and 2D visualization techniques used. We conclude this section with the description of the linking features between the $2 \mathrm{D}$ and $3 \mathrm{D}$ view.

\section{Preprocessing steps}

To extract relevant features from the MR data, several preprocessing steps are required, see also Fig. 2. For this purpose, we used the MASS analytical software package developed at our institute [17]. The endocardial and epicardial contours of the left ventricle and the endocardial contours of the right ventricle are drawn semi-automatically in a shortaxis function scan without contrast since in this type of scan the myocardial boundaries are well visible. These contours are transferred to the LE scan and manually adjusted where necessary to represent the heart shape as best possible. Scar is defined as intensities higher than $35 \%$ of the highest intensity within the myocardium, as visible in the LE scan. The scar is subdivided into gray zone (intensities between 35 and $50 \%$ ) and core infarct (more than 50\%) [8]. For registration purposes, also the ostia of the coronary arteries are indicated in the aorta scan.

For the representation of the endo- and epicardial surfaces, we constructed a mesh from the drawn contours: Sixty-six points per contour were connected to neighboring points in the same and in neighboring slices, forming triangles [18]. When referring to this kind of mesh, we shall call it the 'initial mesh'.

To relate the catheter position - and therefore the voltage measurement positions - to the MR coordinate system, registration is needed. The voltage measurements and the MR data are registered based on the origin of the left main coronary artery, which both are indicated in the MRI and can be indicated with the catheter tip at the start of the ablation procedure. Manual adjustments to this registration can be made where appropriate $[12,19]$.

In summary, the results of the preprocessing we use for our method are: (1) Definition of the myocardium of both the left and right ventricle in the form of contours per slice and the initial meshes of the myocardial surfaces; (2) a scar mask for the MRI slices that indicates the core and gray zone of the scar and (3) the position of the origin of the left main coronary artery.

\section{D visualization techniques}

For the 3D visualization, we developed several multi-modal techniques which can be divided into two categories: The first category makes use of volume rendering of the scar. The second category uses the myocardial surface, extracted from the MRI, to map other parameters on. An overview of the $3 \mathrm{D}$ visualization techniques is visible in Fig. 3. The requirements for the 3D visualizations were providing overview, guiding the catheter and enabling comparison between MR and voltage measurements. First, we will describe the volume rendering techniques and then the MR extracted surface visualizations.

\section{Volume rendering of the scar}

The main contribution of MR to ablation is that the scar locations and characteristics are known prior to the start of the ablation procedure. To present this information in an intuitive way, we made a volume rendering of the scar as can be seen in Fig. 4. The volume rendering was composed by masking the complete volume with the scar mask output of 


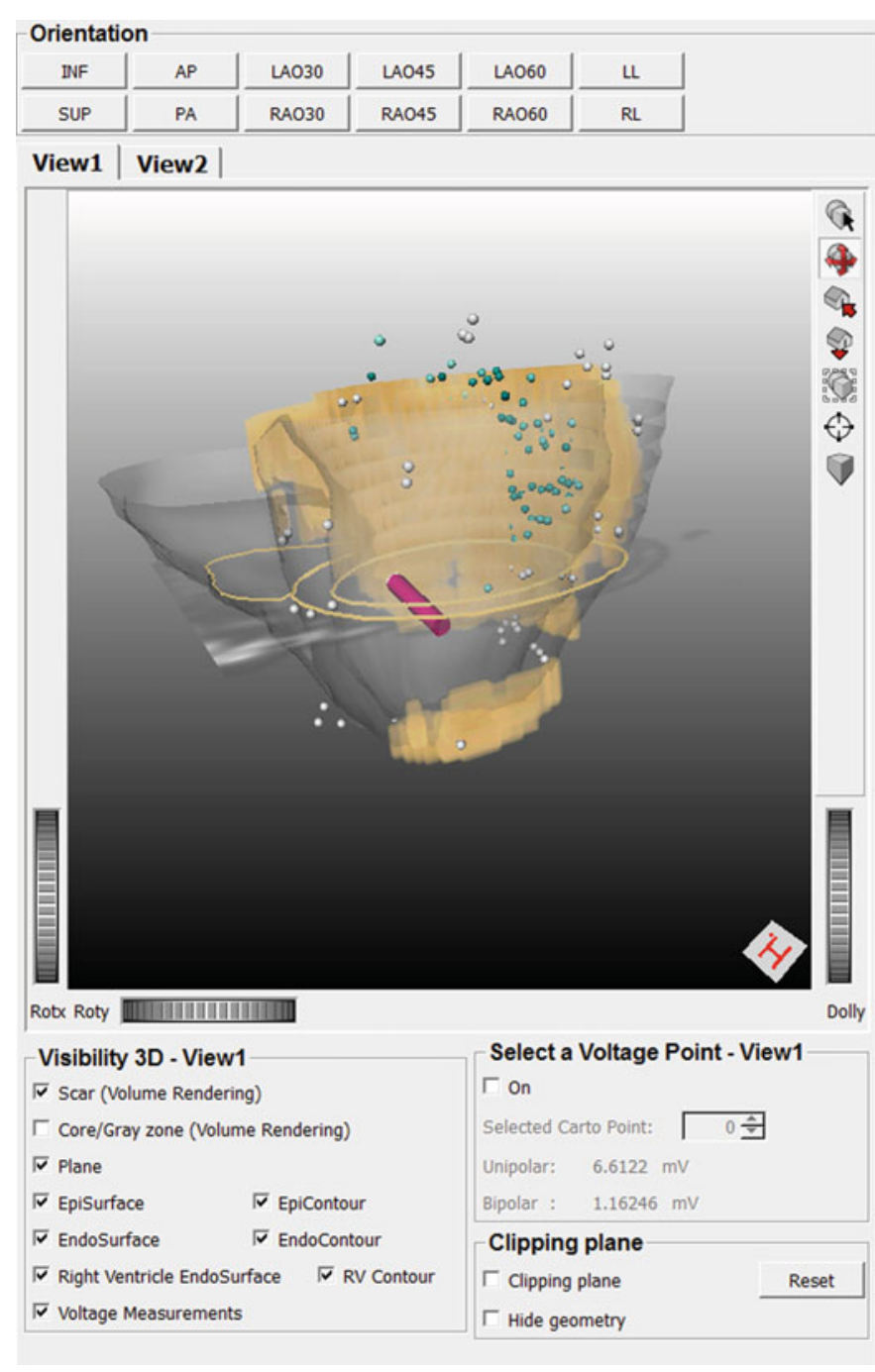

Fig. 1 Overview of the visualization environment. A 3D view and a (three-folded) 2D slice view are combined. At the top, some predefined viewing directions for the $3 \mathrm{D}$ view can be chosen. For the $3 \mathrm{D}$ view, the user can switch between a volume rendering (view1) and a surface rendering (view2) and the context structures can be switched on and off. A clipping plane can be used to clip away obstructing geometries.

the preprocessing stage. We developed two different transfer functions for the volume rendering of the scar tissue. The first one colors the voxels according to their MR signal intensities, in which higher intensities are colored darker. The second one colors the voxels according to scar type, that is, gray or core zone, in which gray zone voxels are colored light and core zone voxels are colored dark. Both transfer functions could be beneficial for the ablation procedure, since it is worth knowing how the intensities are distributed as well as where the gray and core zones are located. The intensitybased transfer function adapts to the data by scaling from zero to the maximum intensity value in the masked data. In Fig. 4, the intensity-based transfer function is used.

For providing context, we added transparent surfaces (opacity $=0.2$ ) representing the endo- and epicardial wall

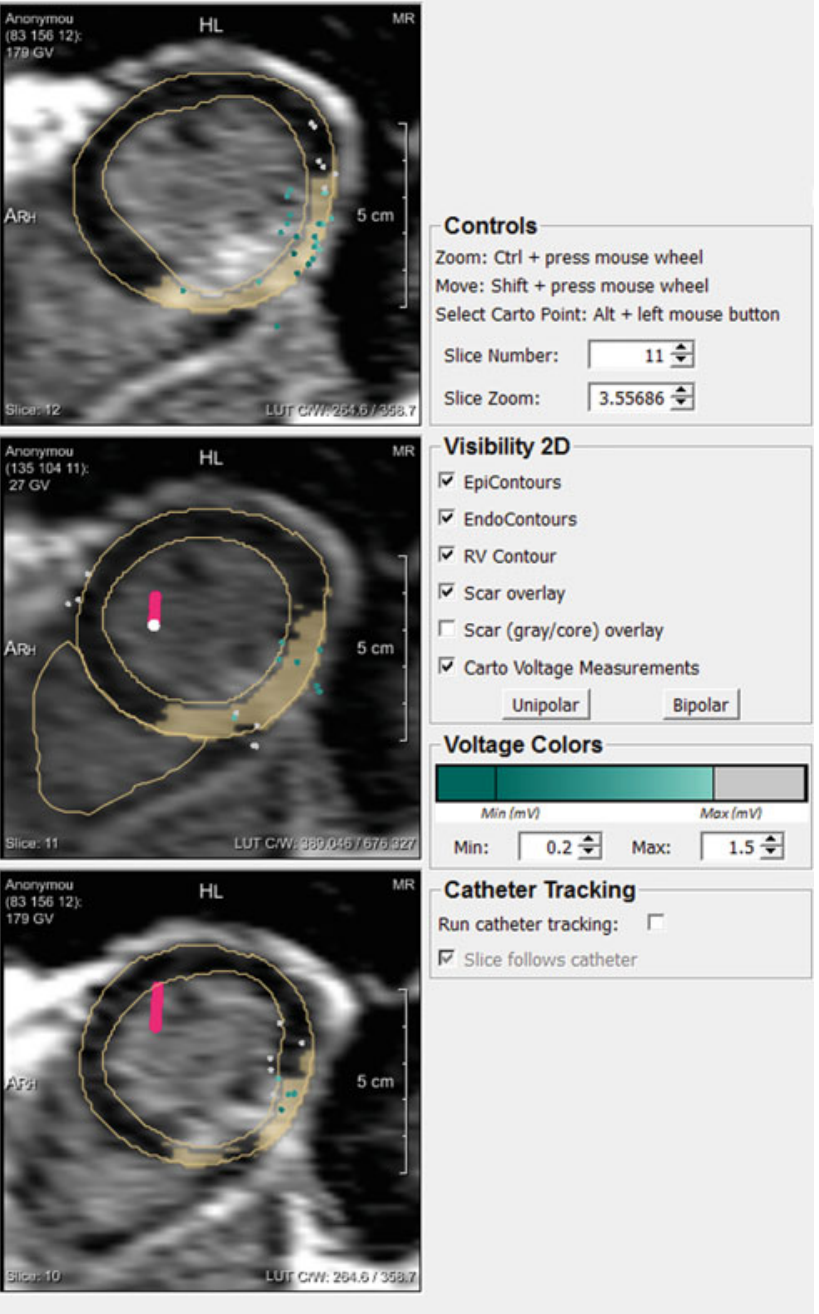

In the 2D view, also the context information can be switched on or off The middle slice is supposed to be the focus slice and is therefore linked to the $3 \mathrm{D}$ view by the linking plane and linking contours, visible in the $3 \mathrm{D}$ view. For the coloring of the voltage measurements, an upper and a lower threshold can be set manually

of the left ventricle. These surfaces are composed from the initial meshes. Also the right ventricle is visualized in this way, providing the user with information about the viewing direction to the left ventricle and the location of the septal wall. The combination of a morphological scar representation and transparent myocardial wall resembles the method of Oeltze et al. [15].

In the same 3D view, the voltage measurements are visualized as small spheres on their location in 3D. The coloring of the spheres is based on the measured voltage: Between a user-defined lower and upper threshold the color changes according to the voltage value, while below the lower threshold and above the higher threshold the color is constant. The user-defined thresholds are there to distinguish the voltages that reflect a possible contribution to a re-entry loop (between 

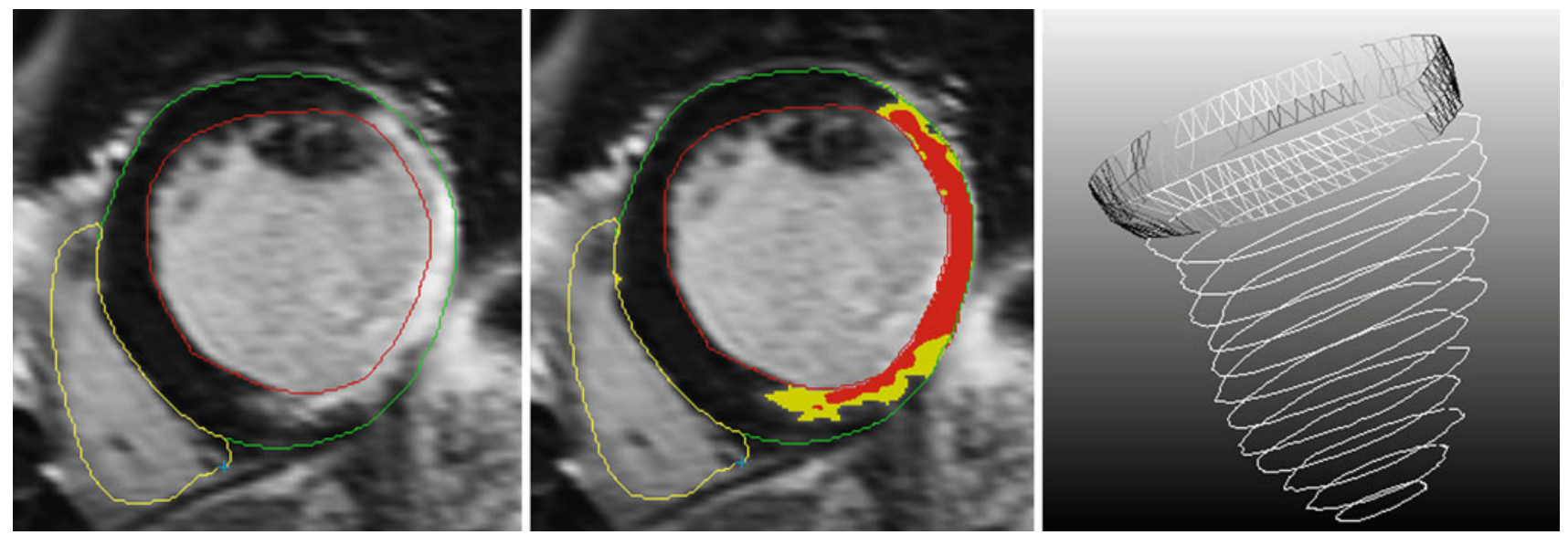

Fig. 2 The preprocessing steps. Left Definition of the contours, Middle Definition of scar (gray zone (in yellow) and core zone (in red)) and Right The composition of the mesh based on the contours in 3D. The

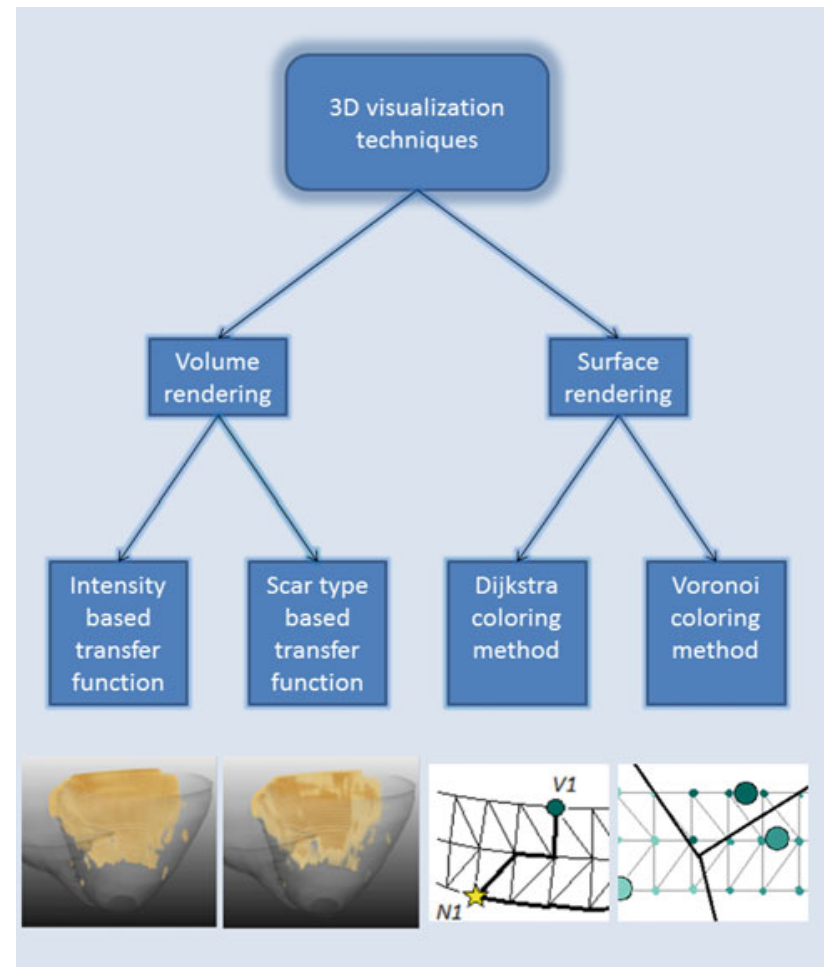

Fig. 3 Overview of the 3D visualization techniques. Two types of volume rendering and two types of surface rendering are developed

the thresholds) from those that are unlikely to contribute (the lower and the higher voltages). By representing these sparse measurements in this way, they can be interpreted well while they do not obstruct the other visualization aspects. The user can choose whether to show the unipolar or the bipolar measured voltage value. By combining the MR information and the voltage measurement in a single view, they can be easily compared. In Fig. 4, the combination of the volume rendering, the transparent surfaces and the voltage measurement representation is illustrated. last described preprocessing step of indicating the position of the origin of the left main coronary artery is not included in this figure

\section{Colored MR extracted anatomy: Voronoi coloring}

Although the voltage measurements are ECG-gated and measurements are obtained during catheter contact with the endocardial or epicardial surface of the heart, the measurement positions do not lie exactly on the MR-derived myocardial wall but are influenced by motion due to beat-to-beat variations and respiratory movements. The $3 \mathrm{D}$ catheter tracking of the system causes measurement inaccuracies as well. However, since all voltage measurements are obtained from the endocardial or epicardial surface of the heart, we strived to give a plausible impression of the voltage distribution by coloring the MR extracted anatomy based on the voltage measurements. In case of endocardial mapping, we colored the endocardial surface mesh, and for epicardial mapping, we colored the epicardial surface mesh. Based on the positions and values of the voltage measurements we calculated a value for every mesh node to color these surfaces. We developed two coloring algorithms: Voronoi coloring and Dijkstra coloring.

The surface mesh that is to be colored is formed from the initial mesh. The mesh resolution was increased by subdividing triangles with long edges. The edge-length threshold was selected, so that at least one additional parallel edge was created between the slices. In this way, the triangles are smaller and better distributed than the triangles of the initial mesh. Also, the subdivided mesh has a higher density, further improving the quality of the mesh visualization.

The Voronoi coloring method colors the MR extracted anatomical mesh by using 3D Voronoi regions [20] created from the voltage measurements. Using this method, mesh nodes are assigned the same color as their nearest mapping point, as calculated by Euclidean distance. Figure 5 shows a 2D equivalent of this Voronoi coloring method. To ensure every voltage measurement contributes to the coloring of the 


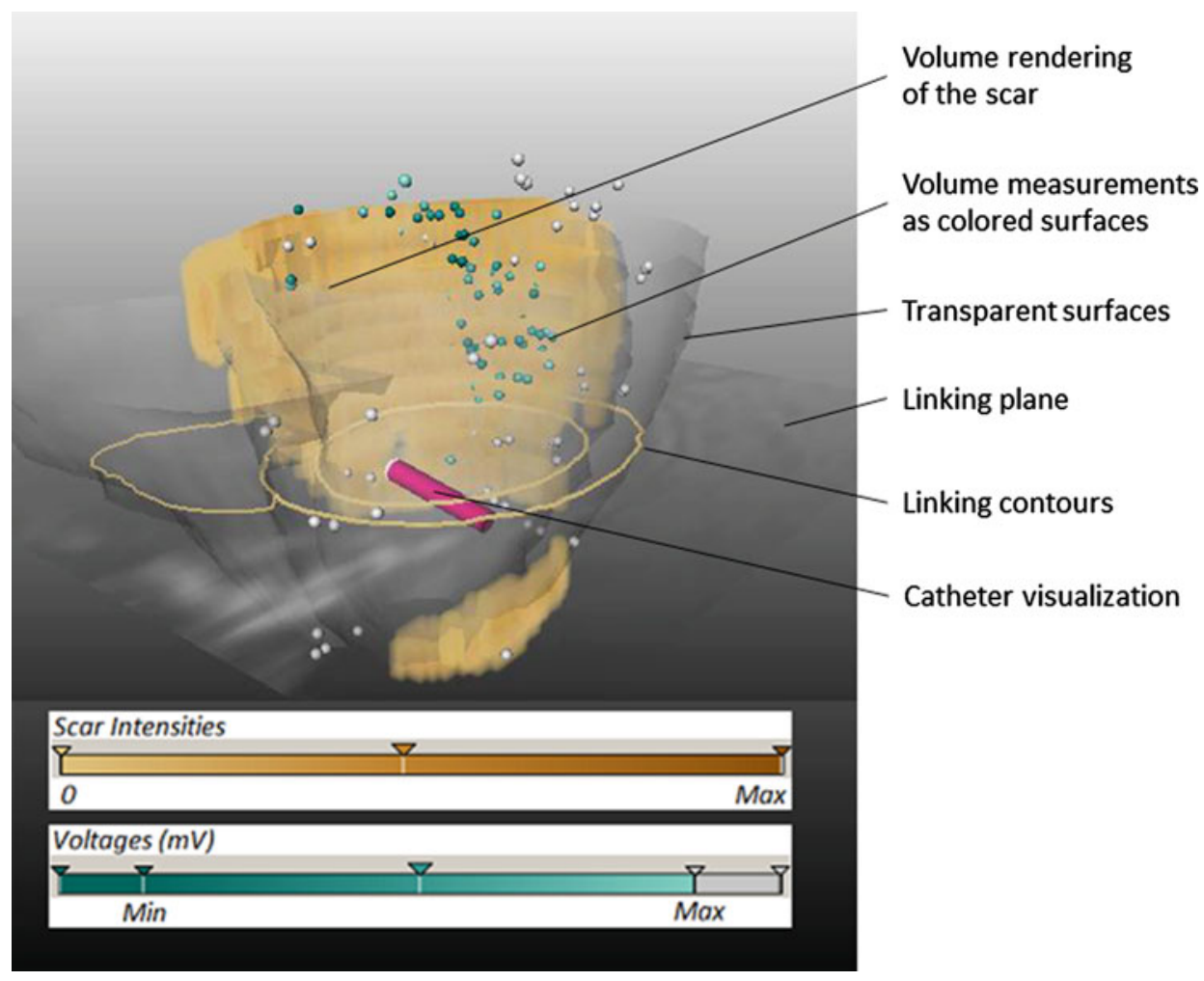

Fig. 4 The volume rendering of the scar is displayed together with the transparent myocardial surfaces. The voltage measurements are represented by the colored spheres. The linking plane, linking contours and the catheter visualization are also visible

surface, first the voltage measurements are projected on their nearest contour. In case of endocardial mapping, the nearest endocardial contour will be chosen, in case of epicardial mapping the measurements will be projected on the nearest epicardial contour. This projection is done in a block-wise manner: First the voltage measurements are projected on the nearest slice, whereafter the nearest contour point within that slice is found. Based on these projected voltage measurements, a 3D Voronoi diagram is created. This 3D Voronoi diagram is then dissected by the anatomical surface mesh, and each mesh node has assigned the value of the Voronoi region that it is positioned in. The main advantage of this method is that it is fast and easy to calculate. A drawback is that it uses Euclidean distances instead of following the shape of the heart wall.

\section{Colored MR-extracted anatomy: Dijkstra coloring}

Our Dijkstra coloring method does take into account the shape of the heart wall. It uses the shortest geodetic path distances as weighting factors for coloring the surface as illustrated in Fig. 6. First, the voltage measurements are projected onto their respective nearest mesh nodes. Then, from every mesh node, the shortest geodetic path to each projected voltage measurement point is calculated using the Dijkstra algorithm [21]. These distances act as weighting factors for the voltage measurement values in the following way:

$n_{i}=\frac{1}{\sum_{j} \frac{1}{d_{i, j}}} \cdot \sum_{j} \frac{v_{j}}{d_{i, j}}$

in which $n_{i}$ is the value of the $i$ th node, $v_{j}$ is the value of the $j$ th voltage measurement and $d_{i, j}$ is the geodetic distance between the $i$ th node and the $j$ th voltage measurement. For nodes that contain one or more distances that are zero, the averaged value of the corresponding voltage measurements is assigned to the node. This prevents blurring of voltage changes at the position of a projected voltage measurement. In Fig. 7, an example of a Dijkstra-colored surface is displayed.

By using this method, the voltage value interpolated at any mesh node contains contributions from all voltage measurements, whereby measurements that are closer contribute more heavily. The main advantage of the use of the Dijkstra algorithm to calculate the distances is that the distances are calculated geodetically and therefore follow the anatomical shape of the myocardium.

\section{Clipping plane}

The purpose of the 3D visualization techniques just described is to provide the user with a global overview of the scar 

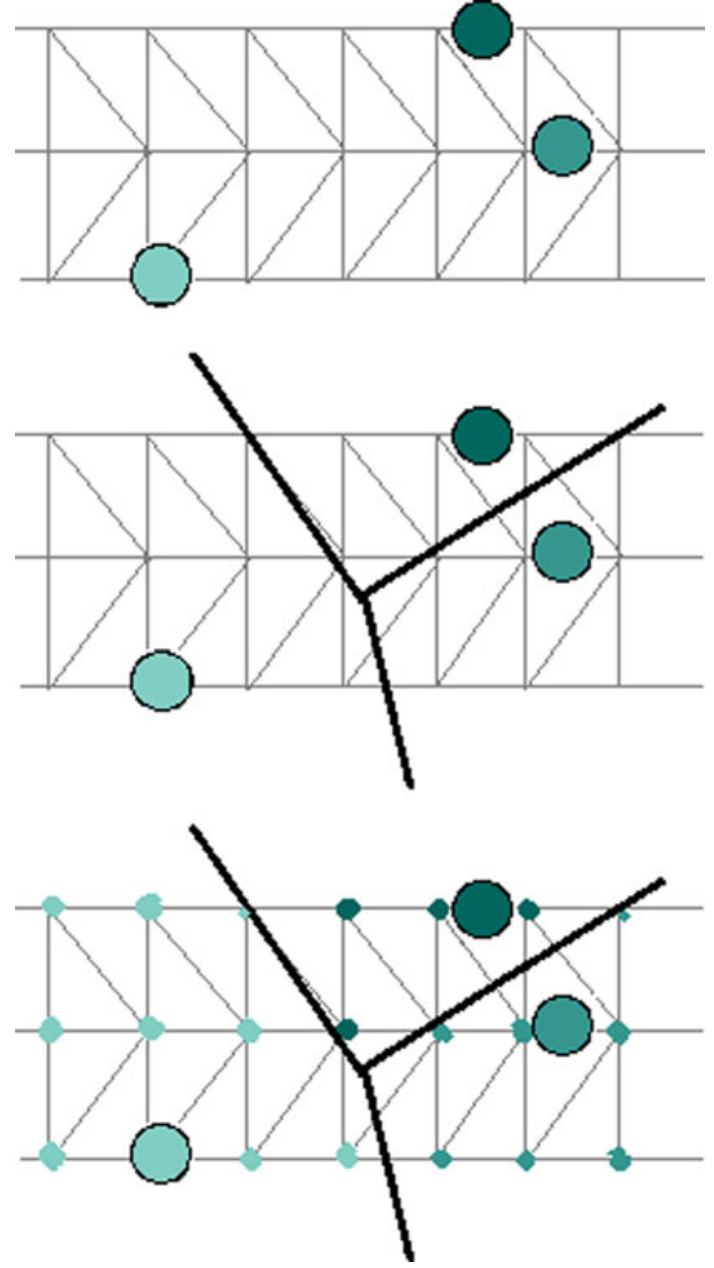

Fig. 5 A 2D example to explain the Voronoi coloring method. Based on the voltage measurement positions, the space is divided into areas according to the Voronoi principle. Every mesh node gets assigned the color of the corresponding voltage measurement

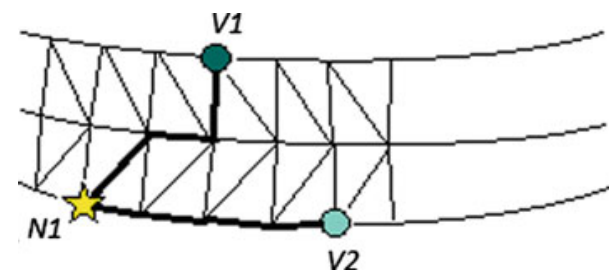

Fig. 6 Explanation of the Dijkstra coloring method. To assign a value to node $\mathrm{Nl}$, the shortest path to all voltage measurements (here $V 1$ and $V 2)$ are calculated by the Dijkstra algorithm [21]. The distances $\left(d_{1,1}\right.$ and $\left.d_{1,2}\right)$ serve as weighting factors for the voltage values $\left(v_{1}\right.$ and $\left.v_{2}\right)$ following Eq. (1) to calculate the value

distribution in the myocardium. The next step in the ablation procedure is to focus on a specific part in the heart. However, it may happen that the visualization obstructs the preferred viewing direction. For those cases, we introduced a clipping plane in the 3D scene, which is used to hide the obstructing, non-interesting parts of the visualization. An example of the

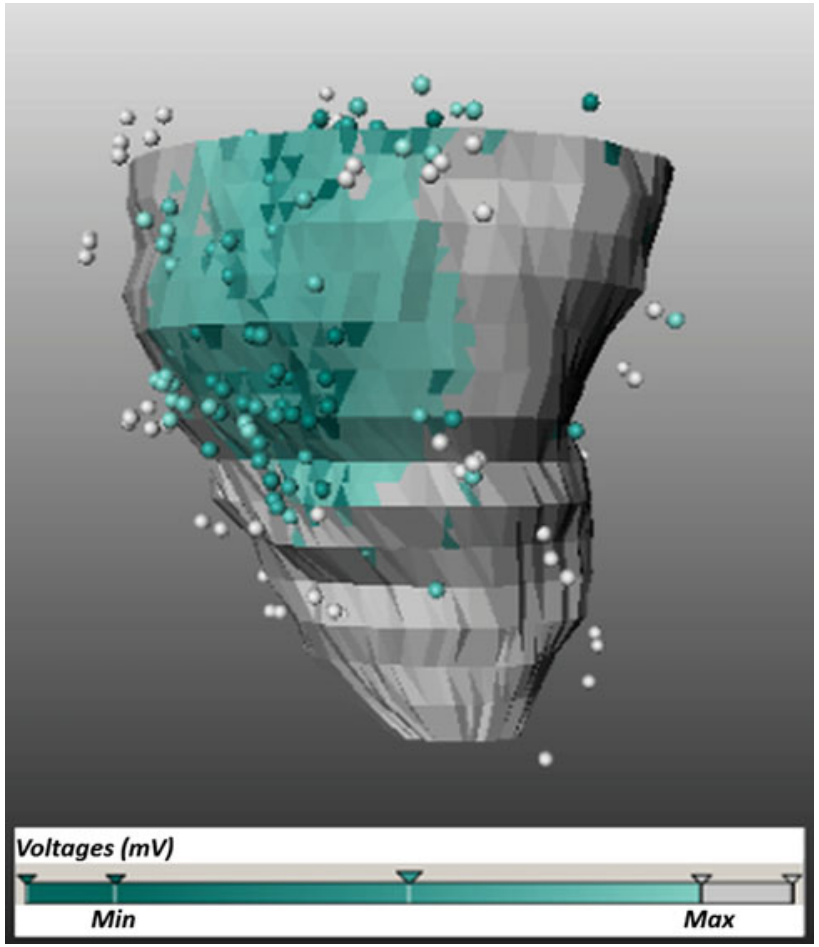

Fig. 7 Myocardial surface colored according to weighted Dijkstra calculations. The original voltage measurements are displayed as well

use of this clipping plane is depicted in Fig. 8. The plane can be placed in every possible position and clips away the information at one side of the plane, while it leaves the other side untouched. In this way, the region of interest can be visualized in the preferred manner and from the preferred direction.

\section{$2 \mathrm{D}$ visualization techniques}

The main advantages of a 2D slice view is that all information from the MRI scan is visible, which makes it a perfect method for assessing detail. Therefore, we developed a 2D slice view in which the original short-axis MRI slices are shown in the conventional orientation. For providing depth feedback, three consecutive slices are visible as can be seen in Fig. 9. The middle view is intended to be the focus slice, while the slices above and below provide insight in the surrounding tissue. To get access to the analysis outcome from the preprocessing steps, the user can choose to show the corresponding left ventricular epicardial contour, left ventricular endocardial contour and right ventricular endocardial contour in the slice as well as a colored overlay of the scar. The voltage measurements can also be displayed on their respective nearest slice. The 2D slice view allows interaction in the sense of scrolling through the slices, setting window level and window width, zooming and panning. It also gives gray value feedback about the voxel the cursor points at. 


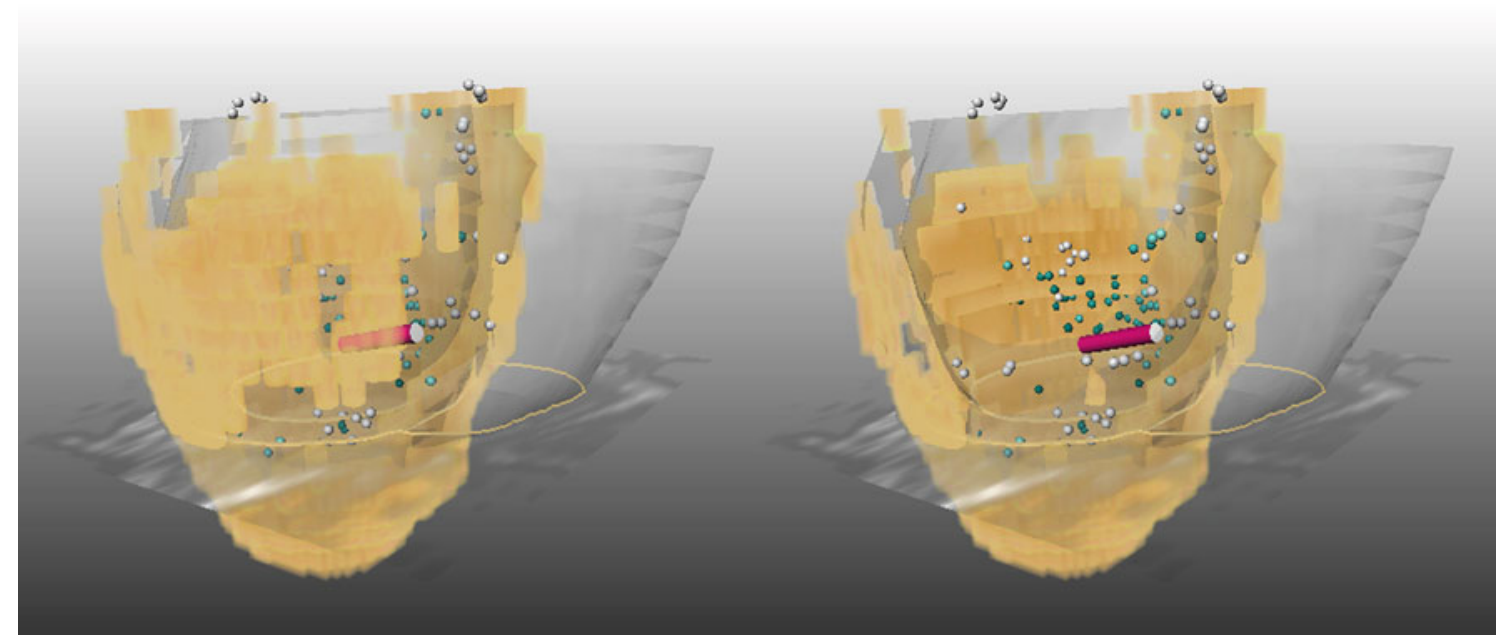

Fig. 8 The use of the clipping plane: For this patient, data scar is obstructing the preferred viewing direction (left). With the clipping plane, this 'front scar' has been clipped away to offer a clear view on the rest of the data (right)

Linking and synchronization of the 3D and 2D view

To take full advantage of the 2D and 3D view simultaneously, and to minimize the mental effort it takes to change the focus from one view to the other, we propose to link and synchronize the two views. Therefore, we extended the multimodal visualization techniques described above with several features in the visualization environment.

\section{Linking plane and linking contours}

Figure 4 shows how we used a plane and contours for linking the $3 \mathrm{D}$ and the $2 \mathrm{D}$ views. To provide the user slice level feedback in the 3D view about which slice is shown in the $2 \mathrm{D}$ view, the focus slice is transparently displayed in 3D. By scrolling through the slices in 2D, the slice in $3 \mathrm{D}$ moves along. We also added the contours to this slice in 3D. The first purpose of these contours is that they provide depth feedback about the position of the scar compared to the heart wall; it is easy to see whether the scar is transmural or not. The second purpose becomes apparent when the transparent slice in 3D causes too much obstruction to the user. The slice can then be turned off while the contours in $3 \mathrm{D}$ remain, and in this way, the slice level feedback is retained with minimal visual obstruction.

\section{Catheter visualization}

For navigation purposes, not only the target area has to be visualized but also feedback on the position of the catheter has to be provided. For that reason, the tip of the catheter is visualized both in the $3 \mathrm{D}$ view and in the $2 \mathrm{D}$ view as can be seen in Figs. 4 and 9. In the 2D slice view, the catheter is visible as the projection of the catheter in the nearest slice.
By visualizing the catheter both in the $2 \mathrm{D}$ and in the $3 \mathrm{D}$ view, the user can intuitively link the visualizations and can choose to navigate based on one or the other or on a combination of both.

\section{Catheter-linked slice level}

To assess the tissue in the 2D slice view while navigating the catheter, we implemented an automatic visualization feature that adapts the slice level to the catheter position. In this way, the focus slice displayed in the 2D slice view follows the catheter by showing its nearest slice. In this way, the tissue that corresponds to the catheter position can be assessed in detail. The catheter linking can also be turned off, which provides the user the ability to inspect the MR data independently from the catheter position.

\section{Corresponding colors}

The color scheme for the volume rendering of the scar and for the voltage measurements is based on the color specifications and designs developed by Cynthia Brewer ${ }^{1}$. The colormap chosen is a diverging colormap of which one side is used for the visualization of the scar while the other side is used for the coloring of the voltage measurements. Theoretically, this colormap is better than the rainbow colormap as it has an intuitive ordering [22]. The selected colormap is also suitable for colorblind users, which always provides good distinction between the voltage measurements and the volume rendering of the scar, as well as an observable ordering of the measured voltages and scar intensities. The colors in the $2 \mathrm{D}$ slice view

\footnotetext{
${ }^{1} \mathrm{http} / / /$ colorbrewer2.org.
} 

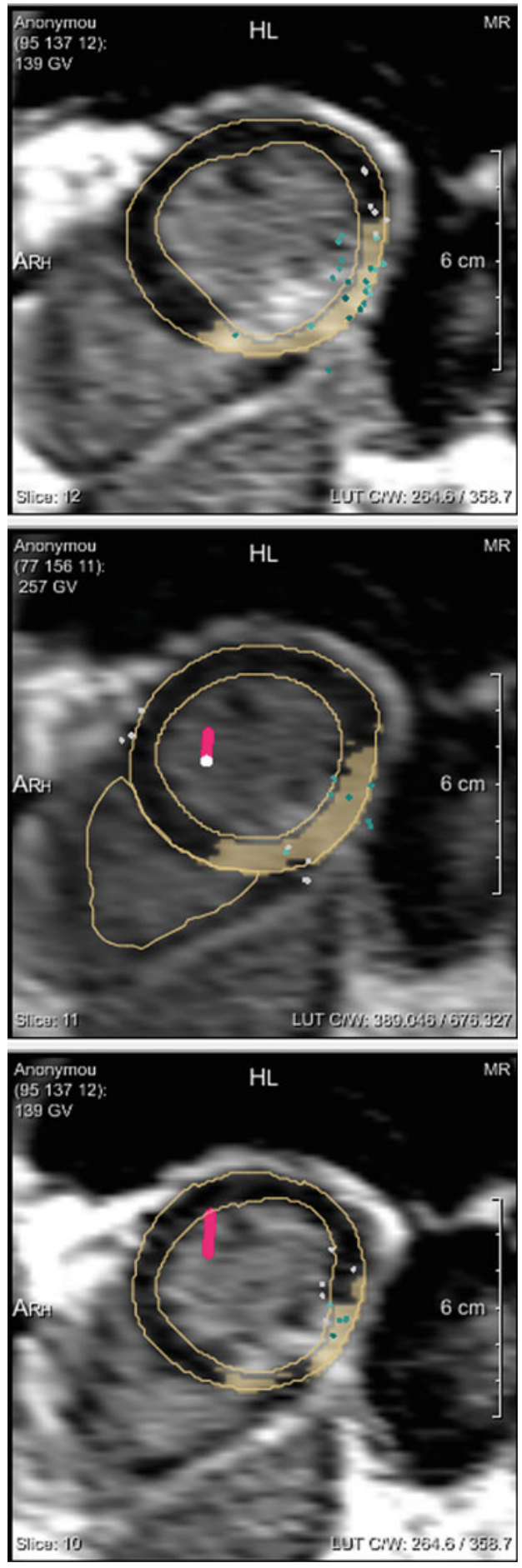

Fig. 9 Three consecutive MRI slices are shown in the 2D slice view. The middle view is supposed to be the focus slice, while the upper and lower view provide insight in the surrounding tissue by showing its neighboring slices. Updating the visible range of slices can be done manually by scrolling or let it automatically follow the catheter tip. For better tissue assessment during the ablation procedure, the myocardial contours are displayed, as well as an overlay of the scar. The voltage measurements are projected onto the nearest slice. The catheter is displayed in red with the white circle as its tip. In the screenshot shown here, the catheter is positioned oblique compared to the slices; therefore, the catheter is displayed in two slices correspond to the colors in the $3 \mathrm{D}$ view, which is helpful to mentally link the two views.

For the evaluation purposes, we also added the more traditional rainbow coloring for the voltage measurements. In Fig. 10, both colormaps are displayed.

\section{Voltage measurement drill-down}

The visualized voltage measurements can be assessed by selecting them individually. The selected point is emphasized both in the 2D and in the 3D view, while the unipolar and bipolar voltage measurements are displayed.

\section{Implementation}

We implemented our method in MeVisLab (MeVis Medical Solutions AG and Fraunhofer MEVIS, Bremen, Germany), a development environment for medical image processing and visualization. The proposed visualization techniques were integrated in a graphical user interface.

For evaluation purposes, we simulated catheter tracking by making use of a Sony PS3 Eye camera and two ARToolkit [23] markers. Tracking was performed using in-house built software based on Processing ${ }^{2}$ and NyARToolkit with multiple marker support ${ }^{3}$. MeVisLab queried the marker transformation matrices in real time from the tracking software via XML-RPC. While moving the marker by hand, the visualized catheter in the interface moves along.

\section{Results}

We evaluated our system on the data of 9 patients. First, we will describe these data, after which the evaluation will follow.

\section{Patient data}

We selected the patient data of 9 patients who were diagnosed with VT, of whom an MR scan was available and who had undergone successful ablation. All patients gave their informed consent for the procedure. No Ethics Committee approval was obtained since the MR scans were part of the routine clinical protocol applied at the Leiden University Medical Center. For every patient, the set of MR scans consist of a function scan (cine scan), an aorta scan and a LE scan. The LE scans were made 15 min after injection of the contrast Gadolinium. In all LE scans, the slices have a thickness of $10 \mathrm{~mm}$ and the centers of the slices have a mutual

\footnotetext{
2 http://processing.org.

${ }^{3}$ http://cpbotha.net/2010/06/05/processing-nyartoolkit-multiplemarker-tracking.
} 


\section{Voltages (mV)}

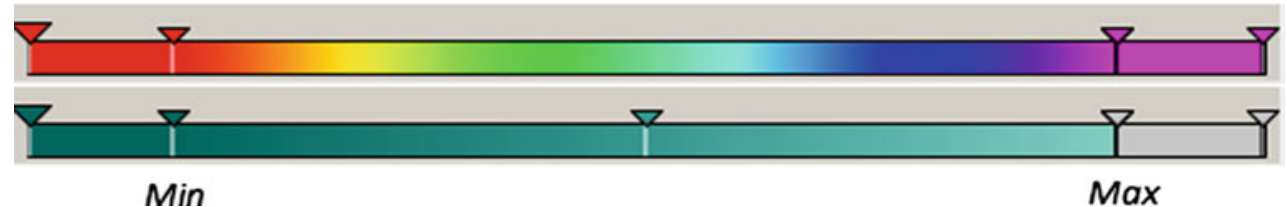

Fig. 10 Two different colormaps for the voltage measurements. The rainbow colormap that is common in clinical practice and the colormap we introduced showing an intuitive ordering. 'Min' indicates the lower

spacing of $5 \mathrm{~mm}$, which means that neighboring slices overlap for $50 \%$. The pixel spacing varied from $1.56 \times 1.56$ to $2.07 \times 2.07 \mathrm{~mm}$ among the selected patients. During the ablation procedures, 8 patients were mapped endocardially and 1 patient epicardially.

\section{High resolution test data}

In order to evaluate the efficacy of our technique on higher resolution data, we created a high resolution version of the MR data of one patient by linearly interpolating the data. The pixel spacing within the slices was halved in both directions to $0.78 \times 0.78 \mathrm{~mm}$ while the spacing between the slices was reduced to $2 \mathrm{~mm}$. The initial meshes were composed in the same way as described in the preprocessing steps section, however, for the colored meshes no further subdivision was done, since the initial mesh was dense enough.

\section{Performance}

Although we performed our method offline, it is worth knowing what the calculation time would be in real time. The MRI data is obtained, analyzed and preprocessed before the ablation procedure starts, while the voltage measurements will be made real time. Our implementation makes use of retrospective data and we added all voltage measurements per patient at once. The calculation time for the different method components were as follows: The 2D visualization techniques, the volume rendering with the voltage measurements as colored spheres and the Voronoi colored surface were all instantly available. Thus, these parts can also be used in a real-time system. The Dijkstra colored surface in our implementation took for a mesh with a typical size less than 4 seconds per voltage measurement point. This algorithm would need optimization to reduce this computation time to make it useful in a real-time system.

\section{Evaluation}

To evaluate our method, we performed a case study evaluation, following the guidelines for case study research set out by Yin [24]. By means of several propositions, we aimed to threshold, while 'Max' corresponds with the upper threshold, both to be set by the user

answer the main study question, which was defined as: "How do the proposed multi-modal visualization techniques offer better catheter guidance and tissue assessment to the user who performs the VT ablation?" As the case, we simulated the visual environment of an ablation procedure containing the proposed multi-modal visualization techniques. During this simulation, we observed and evaluated the system based primarily on the experience of our third author, a cardiologist and a clinical expert in VT ablations. The author has extensive experience with the 3D CARTO mapping system (Biosense Webster, Cordis-Webster, Johnson \& Johnson, Diamond Bar, CA, USA) and with the 3D Ensite NavX mapping system (St Jude Medical, St Paul, MN, USA). In the following, we comment in a structured way on the different elements of the system from a clinical point of view, also giving arguments for preferences in cases where multiple options are available. The clinical author was involved in the requirements specification phase, gave feedback on early prototypes of the system and was responsible for this evaluation.

\section{Volume rendering of the scar in $3 D$}

The volume rendering offers better insight in the morphology of the scar than the scar transmurality maps. Furthermore, it was suggested to add the possibility to make the scar transparent, which facilitates catheter navigation without the need of fluoroscopy at areas where the catheter is obscured by dense scar tissue.

\section{Transparent heart wall surface as context visualization}

Context visualization in the neighborhood of the scar is important to interpret the area (i.e. the relative size and location of scar tissue), but context structures that are located away from the scar provide no additive information and should be turned off. Therefore, it is not necessary to show the whole surface of the left ventricle, although due to the transparency it is not an obstructing feature. For the right ventricle, the same holds: The insertion of the right ventricle can vary between patients, and its location is useful to display if scar is located in the interventricular septum. Based on that information, the user can choose quickly how it can 
be reached best; from the left or the right ventricle. In contrast, for scar located in the lateral wall, the exact location of the right ventricle insertion is not of interest. The context visualization possibilities could be expanded by showing anatomical landmarks like aorta, atria and papillary muscles.

\section{Transfer function for the volume rendering}

The type-based transfer function was considered more helpful than the intensity-based transfer function in providing information about the scar characteristics (i.e. gray zone versus core infarct). The pre-defined cut-off value of the intensities that splits the scar in core infarct and gray zone makes the data easier to interpret than the slightly varying color of the intensities. It has been further suggested to define more intensity categories and visualize the isocontours of the selected signal intensities to provide insight in abrupt changes due to clear gradient visibility.

\section{Clipping plane}

The clipping plane function is a useful feature if the scar area of interest is obscured by scar located at the opposite wall. In these cases, the region of interest can easily be brought into view and be viewed from the preferred viewing direction. Improvement of the clipping plane function would include introducing predefined planes or predefined plane axes based on anatomical structures in the heart, in order to limit the amount of degrees of freedom of the plane. This would facilitate the communication between the operator and the assistant controlling the visual environment, as the preferred clipping plane orientation could be standardized.

\section{$2 D$ slice view}

The 2D slice view provides detailed information from the MR data to assess the myocardial tissue properties. The most useful application of the 2D slice view is the ability to reassess the tissue during the ablation procedure when the catheter is in contact with the site of interest. Electroanatomical voltage information can be compared with MRI-derived scar information. For instance, small spots in the MRI may be defined in the preprocessing procedure as normal tissue but when abnormal voltages are measured in that area the user can easily compare the corresponding original MRI slices and reassess the tissue. Another advantage of the MRI slices is that it is easy to see where surrounding structures are located.

\section{$2 D$ depth feedback by the 3 consecutive slices}

The display of 3 consecutive slices in the 2D view provides extra depth feedback to the user and insight in the surrounding tissue visible in the focus slice. It is convenient that there are more slices visible simultaneously; it can help the user to observe the extension of the scar through the slices without scrolling back and forth through the stack of slices. The scrolling during the procedure has to be done by the assistant, which makes it a cumbersome operation.

\section{Contours and scar overlay in the $2 D$ view}

These features are helpful to locate the scar and assess the tissue, because it helps to clearly visualize the transmurality of the scar and the distance of the scar from the endo- and epicardial surface. It is a helpful feature to focus immediately on the parts that are of interest because the user does not have to redefine mentally the contours and the scar.

\section{The $3 D$ representation of the voltage measurements in the form of colored spheres}

The spheres were difficult to localize and it was not clear which points are likely to be "inner" points acquired without wall contact. It was also difficult to easily see steep gradients (abrupt changes) in the voltage measurements because the values have to be interpolated mentally. It was also mentioned that bipolar voltage measurements are no actual point measurements as the catheter tip covers a region with a size of several square millimeters. Therefore, interpolated colors on a surface would be a more realistic representation, although a way has to be found to represent simultaneously the MRI data.

\section{Visibility of the multiple modalities}

The visualizations of the MR-defined scar and of the voltage measurement points do not obstruct each other. Both modalities are visible the same time.

\section{Relation between the multiple modalities}

It was difficult to relate the MR to the voltage measurements, as the voltage measurements were difficult to interpret due to their representation as colored spheres.

\section{$3 D$ view as a whole}

The 3D view provides a global overview of the scar location and helps to navigate the catheter to it. Although the details of the MRI are lost, the 3D view offers also information that is difficult to extract from the $2 \mathrm{D}$ view. It provides a 3D representation of the scar in the heart wall, which facilitates the imagination of the real 3D anatomy. It was also pointed out that after having chosen a region of interest it would not be necessary anymore to see the whole heart but rather focus on the area and structures in the close vicinity of that region. 
However, in particular for epicardial mapping, it is important to visualize the whole heart, including the atria and also related structures like the aorta and pulmonary artery as anatomical landmarks.

\section{Projection of the voltage measurements on the MR-extracted surface}

It is preferable to project the voltage measurements on the MR-extracted surface, since the voltage measurements are measured on the surface of the heart wall and both the MR and the voltage measurements are ECG-gated. To improve this feature, the papillary muscles should be taken into account and voltage information should be projected on these intracavitary structures. It should also be possible to remove incorrect measurements manually since those measurements will have negative influence on the coloring of the surface.

\section{The voltage measurements in $2 D$}

This would be an interesting feature for post-processing, but these are less important during the ablation procedure. For the user it is not of interest to see the voltage measurements in the $2 \mathrm{D}$ view, also because points are not a realistic representation of the measurements. The projection of the tip of the catheter on the slices, however, is sufficient to assess the tissue in an area of interest. To improve the usability of the 2D view it would be helpful to place tags on user-defined spots, which are visible both in 2D and in 3D. In that way, the user can indicate important areas during the mapping procedure.

\section{$2 D / 3 D$ linking}

The transparent slice and the corresponding contours in 3D are helpful to intuitively link the 3D view with the 2D slice view and to give feedback about the slice level in the $2 \mathrm{D}$ view. The linking contours in 3D are slightly better for that purpose than the transparent slice, since the contours are already effective and do not obstruct the rest of the visualization. From the contours, it is directly clear where the focus slice is located and thus it is an effective linking feature. The distribution and position of the scar in the heart wall can best be assessed in the 2D view, and with the use of the linking contours in $3 \mathrm{D}$, it is easy to link the $2 \mathrm{D}$ view to the position in $3 \mathrm{D}$.

\section{Catheter visualization}

The catheter being visible in both the $3 \mathrm{D}$ and $2 \mathrm{D}$ views would help the user to link the two views and to localize the catheter. It is convenient that the catheter is visible both in $3 \mathrm{D}$ and in 2D.

\section{Voltage colormap}

The color coding of the voltage map already used in clinical practice for many years (rainbow coloring) seems clearer than the new color coding we proposed. Clinicians are used to and expect the rainbow colormap, as it is applied in all available and currently used 3D mapping systems.

\section{The 'slice follows catheter' setting}

This option is useful to inspect directly the corresponding MR slice while navigating with the catheter, especially while making little movements with the catheter. The ability to switch it off is appropriate if large catheter movements are performed, for example, to quickly acquire the anatomy, since in that case the slice will move too quickly to be inspected.

\section{Dataset in high resolution (by interpolation of the original MR scan)}

The smaller voxel size results in visualizations that appear smoother and better-looking. However, interpolated values could give false confidence. The best alternative for this interpolated high resolution data would be an MRI scanned in a higher resolution.

\section{Study conclusions}

From a clinical point of view, the proposed techniques for presenting MRI data during the ablation have the potential to improve the mapping procedure. During the interview, also additional, practical suggestions for improvement were made, which made the case study meeting a fruitful interview for further experiments.

Based on this evaluation, we conclude that the proposed use of a linked 2D and 3D view can facilitate the VT mapping procedure. In the 3D view, a good overview of the scar locations can be obtained for catheter guidance, while in the 2D view, the tissue characteristics can be assessed in detail. The linking of the two views by the linking contours causes reinforcement of the views, which makes it an effective entity. However, the visualization of the voltage measurements in the multi-modal representation has to be adjusted to be able to better interpret the measurements.

\section{Limitations}

Although the main message of the evaluation is that the proposed approach of representing MRI during ablation procedures is promising, this case study also exposed some limitations. 
In clinical practice, for example, in the CARTO software, rainbow colormaps have been in use for many years to color code voltage measurements. However, it has been pointed out a number of times in literature, recently for example by Borland and Taylor [22], that rainbow colormaps have at least two serious problems: (1) There is no inherent ordering, that is, one does not know whether green comes before or after yellow and (2) they are not perceptually linear, that is, colors that to humans appear to be different are close to each other on the rainbow colormap and vice versa. In spite of these issues, clinical familiarity with the rainbow colormap complicates the acceptance of new and improved colormaps that address the abovementioned issues, a phenomenon that was confirmed by our observations implementing the prototype described in this paper.

To evaluate which surface coloring method represents best possible successful ablation sites, a quantitative evaluation technique is needed. Through the case study, we evaluated only the idea of colored surfaces. Other evaluation techniques have to demonstrate whether the Voronoi or the Dijkstra coloring method is better.

Due to time limits and the fact that only one operator is performing VT ablation procedures in our hospital, we were not able to include more than one user in the evaluation. We are aware of the limitations and the possible bias that this entails, though this is justifiable for this early stage of investigation. Additionally, VT ablation is only performed by a small number of experts.

During the evaluation, it was also mentioned that caution should be exercised when the heart rate, heart volume and the presence of arrhythmia is significantly different during mapping and ablation than during the acquisition of the MRI, because this may cause a change of the heart position and shape. The best way to integrate MRI in the ablation procedure would be the use of real-time MRI: that would be most reliable since the patient is in the same condition during MRI and voltage measurements. That, however, is beyond the scope of this study.

\section{Discussion and future work}

The major part of the visualization system we propose was assessed positively, however, not every visualization aspect turned out the way it was intended. The most notable part is that the voltage measurements in the representation of the colored spheres did not provide sufficient detail for their effective interpretation. Furthermore, a useful colormap should be developed for the voltage measurement values. It should address the conflict between a known, recognizable colormap and a perceptually optimized colormap. A good colormap will contribute to more safety for the patient and greater ease of use for the cardiologist. However, these should be developed in close collaboration with clinicians.
What also became clear is that providing context by the visualization of neighboring structures is important for the usability and interpretation of the MRI data. The ability to switch particular structures on or off is important, since it is patient and scar dependent what the useful context structures are. It even depends on the part of scar the user focuses on, which can vary during the procedure. Future work can be done on the extension of the structures to display, like papillary muscles, atria and aorta.

Another way to display more relevant information is the use of long-axis views of the MRI. Especially around the very basal and apical level of the left ventricle, long-axis views can provide useful information. Adding information from long-axis views, or even better, information from true 3D isotropic MR imaging could be a future improvement of the system.

The question 'what surface coloring is the best' was beyond the scope of this study but it still is a pressing one. Comparing retrospectively several surface coloring methods to successful ablation sites has to show which surface coloring is a reliable representation and thus would be best to use.

To make this visualization system ready to be used in the clinic, the calculations for new voltage measurements have to be performed real-time. It appeared that only the Dijkstra surface coloring needs optimization for this purpose. Since we computed this algorithm in a brute force way, there are several possibilities to reduce its calculation time. For instance by using a fast marching method [25] or by calculating only relevant distances. We expect that by optimization of this method, the calculation time per voltage measurement can be reduced to less than half a second.

We did not take into account any measurement of registration error. A registration error of several millimeters is common with this registration approach [12,19], and these distances could influence the measurement projection and surface coloring. However, we expect that registration inaccuracies will not cause major changes in the visualization given the MRI spacing and mesh density. Though in future versions, registration error feedback should be included to show the user the accuracy of the visualization.

After improving the proposed system according to the suggestions listed here, it can be made available during catheter ablation procedures. The research documented in this paper gives clear guidelines as to how current ablation systems could be further improved.

\section{Conclusions}

In this paper, we have presented the first practical investigation of the integrated visualization of MRI data and voltage measurements in order to improve VT ablation procedures. 
The proposed method links a 3D and a 2D multi-modal view containing MRI data and voltage measurements. The 3D view serves for catheter guidance and provides good overview over the heart while the $2 \mathrm{D}$ view offers the desired amount of detail to assess the tissue. The linking of the views makes it convenient to use both views simultaneously.

Based on the preprocessing results in which the myocardial contours and the scar have been indicated, we developed for the $3 \mathrm{D}$ view a volume rendering of the scar with additional context visualizations. The voltage measurements are indicated as little colored spheres. Another 3D visualization technique we developed contains an MR extracted surface colored according voltage measurements. For the 2D view, we developed a display of three consecutive slices in which the preprocessing results can be showed. To link the 3D and $2 \mathrm{D}$ view, we developed a linking plane and linking contours, which show in the $3 \mathrm{D}$ view the slice level of the $2 \mathrm{D}$ focus slice. The catheter is also visible in both views and the colors correspond in both views.

To evaluate this system, we performed a case study in which we simulated a VT mapping and ablation procedure while we held a focused interview with an expert user. The visualization method we proposed and developed was assessed positively, especially the volume rendering of the scar with the transparent context visualizations, the overlay of contours and scar in the 2D view and the linking contours. Future work has to be done for the visualization of the voltage measurements to show its full advantage in clinical practice.

\section{Conflict of interest None.}

Open Access This article is distributed under the terms of the Creative Commons Attribution License which permits any use, distribution, and reproduction in any medium, provided the original author(s) and the source are credited.

\section{References}

1. Aliot EM, Stevenson WG, Almendral-Garrote JM, Bogun F, Calkins CH, Delacretaz E, Della Bella P, Hindricks G, Jaïs P, Josephson ME, Kautzner J, Kay GN, Kuck K-H, Lerman BB, Marchlinski F, Reddy V, Schalij M-J, Schilling R, Soejima K, Wilber D (2009) EHRA/HRS expert consensus on catheter ablation of ventricular arrhythmias. Heart Rhythm 6(6):886-933

2. Bogun FM, Desjardins B, Good E, Gupta S, Crawford T, Oral H, Ebinger M, Pelosi F, Chugh A, Jongnarangsin K, Morady F (2009) Delayed-enhanced magnetic resonance imaging in nonischemic cardiomyopathy: utility for identifying the ventricular arrhythmia substrate. J Am Coll Cardiol 53(13):1138-1145

3. Desjardins B, Crawford T, Good E, Oral H, Chugh A, Pelosi F, Morady F, Bogun F (2009) Infarct architecture and characteristics on delayed enhanced magnetic resonance imaging and electroanatomic mapping in patients with postinfarction ventricular arrhythmia. Heart Rhythm 6(5):644-651
4. Raymond J-M, Sacher F, Winslow R, Tedrow U, Stevenson WG (2009) Catheter ablation for scar-related ventricular tachycardias. Curr Probl Cardiol 34:225-270

5. Haqqani HM, Marchlinski FE (2009) Electrophysiologic substrate underlying postinfarction ventricular tachycardia: characterization and role in catheter ablation. Heart Rhythm 6:S70-S76

6. Eckardt L, Breithardt G (2009) Catheter ablation of ventricular tachycardia. From indication to three-dimensional mapping technology. Herz 34(3):187-196

7. Heatlie GJ, Pointon K (2004) Cardiac magnetic resonance imaging. Postgrad Med J 80(939):19-22

8. Roes SD, Borleffs JW, van der Geest RJ, Westenberg JJM, Marsan NA, Kaandorp TAM, Reiber JHC, Zeppenfeld K, Lamb HJ, de Roos A, Schalij MJ, Bax JJ (2009) Infarct tissue heterogeneity assessed with contrast-enhanced MRI predicts spontaneous ventricular arrhythmia in patients with ischemic cardiomyopathy and implantable cardioverter-defibrillator. Circ Cardiovasc Imaging 2:183-190

9. Schmidt A, Azevedo CF, Cheng A, Gupta SN, Bluemke DA, Foo TK, Gerstenblith G, Weiss RG, Marbán E, Tomaselli GF, Lima JAC, Wu KC (2007) Infarct tissue heterogeneity by magnetic resonance imaging identifies enhanced cardiac arrhythmia susceptibility in patients with left ventricular dysfunction. Circulation 115(15):2006-2014

10. Yan AT, Shayne AJ, Brown KA, Gupta SN, Chan CW, Luu TM, Di Carli MF, Reynolds HG, Stevenson WG, Kwong RY (2006) Characterization of the peri-infarct zone by contrast-enhanced cardiac magnetic resonance imaging is a powerful predictor of post-myocardial infarction mortality. Circulation 114(1):32-39

11. Dickfeld T, Tian J, Ahmad G, Jimenez A, Turgeman A, Kuk R, Peters M, Saliaris A, Saba M, Shorofsky S, Jeudy J (2011) MRIGuided ventricular tachycardia ablation: integration of late gadolinium-enhanced 3D scar in patients with implantable cardioverter-defibrillators. Circ Arrhythmia Electrophysiol 4(2):172184

12. Wijnmaalen AP, van der Geest RJ, van Huls van Taxis CFB, Siebelink H-MJ, Kroft LJM, Bax JJ, Reiber JHC, Schalij MJ, Zeppenfeld K (2011) Head-to-head comparison of contrastenhanced magnetic resonance imaging and electroanatomical voltage mapping to assess post-infarct scar characteristics in patients with ventricular tachycardias: real-time image integration and reversed registration. Eur Heart J 32(1):104-114

13. Codreanu A, Odille F, Aliot E, Marie P-Y, Magnin-Poull I, Andronache M, Mandry D, Djaballah W, Régent D, Felblinger J, de Chillou C (2008) Electroanatomic characterization of postinfarct scars: comparison with 3-dimensional myocardial scar reconstruction based on magnetic resonance imaging. J Am Coll Cardiol 52(10):839-842

14. Termeer M, Oliván Bescós J, Breeuwer M, Vilanova A, Gerritsen F, Gröller E (2007) CoViCAD: comprehensive visualization of coronary artery disease. IEEE Trans Vis Comput Graph 13(6):16321639

15. Oeltze S, Hennemuth A, Glaßer S, Kühnel C, Preim B (2008) Glyph-based visualization of myocardial perfusion data and enhancement with contractility and viability information. In: Spring Eurograph Visual Computer Biomedicine

16. Termeer MA (2009) Comprehensive visualization of cardiac MRI data. Vienna University of Technology

17. van der Geest R, Buller V, Jansen E, Lamb H, Baur L, van der Wall E, de Roos A, Reiber J (1997) Comparison between manual and automated analysis of left ventricular volume parameters from short axis MR images. J Comput Assist Tomogr 21:756-765

18. Drebin RA, Carpenter L, Hanrahan P (1988) Volume rendering. In: Proceedings of SIGGRAPH'88, computer graphics, vol 22, no. 4 , pp 65-74 
19. Tao Q, Milles J, van Huls van Taxis C, Lamb HJ, Reiber JHC, Zeppenfeld K, van der Geest RJ (2012) Toward magnetic resonance-guided electroanatomical voltage mapping for catheter ablation of scar-related ventricular tachycardia: a comparison of registration methods. J Cardiovasc Electr 23(1):74-80

20. Aurenhammer F (1991) Voronoi diagrams - a survey of a fundamental geometric data structure. ACM Cumput Surv 23(3):345405

21. Dijkstra EW (1959) A note on two problems in connexion with graphs. Numer Math 1(1):269-271

22. Borland D, Taylor RM (2007) Rainbow color map (still) considered harmful. IEEE Comput Graph. IEEE Computer Society, Los Alamitos, vol 27, pp 14-17
23. Kato H, Billinghurst M (1999) Marker tracking and HMD calibration for a video-based augmented reality conferencing system. In: International workshop on augmented reality (IWAR'99) IEEE Computer Society, vol 85

24. Yin RK (2009) Case study research: design and methods. Sage, Beverly Hills

25. Kimmel R, Sethian JA (1998) Computing geodesic paths on manifolds. Proc Natl Acad Sci USA 95(15):8431-8435 